\title{
Unexpected news in renal fibrosis
}

\author{
Juan A. Oliver \\ Department of Medicine, Columbia University, New York, New York, USA \\ J. Clin. Invest. 110:1763-1764 (2002). doi:10.1172/JCI200217399.
}

A striking characteristic of many diseases of the kidney is their ability to induce decreases in function that may initially be modest but that inexorably progress until all function is lost. The anatomical correlate of this evolution is a relentless substitution of renal parenchymal cells by fibrotic tissue. Fibrosis had long been recognized as a prominent feature of diseased kidneys, but nephrology's intellectual landscape had long focused on the widely variable and aesthetically alluring glomerular changes caused by disease. A seismic change was initiated by two important discoveries that initially concerned glomerular diseases but have proven to have much wider implications in our understanding of the mechanisms responsible for the inexorable evolution of many renal diseases. First, the hypothesis by Brenner and collaborators that increases in glomerular pressures and flows initiate progressive loss of renal function led to the discovery that inhibitors of the renin-angiotensin system retard renal-function loss and ameliorate renal fibrosis $(1,2)$. Second, Border and colleagues discovered that TGF- $\beta 1$ is a key mediator of renal fibrogenesis $(3,4)$. This work not only resulted in substantial advances in patient care (2) but also initiated a veritable explosion of research aimed at elucidating the mechanisms regulating

\footnotetext{
Address correspondence to: Juan A. Oliver, College of Physicians and Surgeons, Columbia University, 630 West 168th Street, New York, New York 10032, USA.

Phone: (212) 305-6938; Fax: (212) 305-3475;

E-mail: jao7@columbia.edu.

Conflict of interest: The author has declared that no conflict of interest exists.

Nonstandard abbreviations used: unilateral ureteral obstruction (UUO); angiotensin receptor type 1a (Agtr1a); green fluorescent protein (GFP).
}

renal fibrogenesis. Elegant experimental design has attracted many researchers to a model of fibrosis resulting from unilateral ureteral obstruction (UUO), but many other types of renal diseases have also been studied. As it is likely that different pathogenetic mechanisms are activated in different types of renal fibrotic diseases, generalizations must be made cautiously. However, several observations appear to be well established and to apply to most models: (a) the critical role of TGF- $\beta 1$; (b) the profibrotic action of angiotensin (at least in part mediated by TGF- $\beta 1$ ); (c) the antifibrotic effect of inhibitors of the renin-angiotensin system; and (d) the recent demonstration that many renal fibroblasts derive from conversion of renal epithelial cells to mesenchymal cells (5), a reversal of the process that normally occurs during renal development (6). It must be noted, however, that a small number of renal fibroblasts migrate to the kidney from the bone marrow.

\section{Macrophages and renal fibrosis}

Macrophages have critical roles in wound healing, and a vast amount of literature suggests that they promote atherogenesis. Perhaps for these reasons, and because in many conditions the extent of renal fibrotic tissue and the number of infiltrating macrophages change in parallel, these cells have long been suspected to contribute to loss of renal function and renal fibrosis. Support for this hypothesis has been found in renal diseases of animals depleted of macrophages (7) and in animals lacking genes that encode proteins important in macrophage recruitment (8-10). The mechanism(s) whereby macrophages may facilitate renal fibrogenesis is undefined, and the myriad functions of which macrophages appear to be capable suggest that their role in this process may well be very complex. Indeed, in this issue of the JCI, Nishida et al. (11) present evidence that infiltrating macrophages in the kidney may play a beneficial antifibrotic role that, surprisingly, requires the action of angiotensin.

\section{Angiotensin regulation of macrophage function}

It has previously been shown that mice lacking the gene for the angiotensin receptor type 1a (Agtr1a, the predominant isoform in mice) develop less renal fibrosis with lower macrophage infiltration than wildtype mice during UUO (12), findings consistent with the well-known antifibrotic effect of inhibitors of the renin-angiotensin system and with work done with other genetic models with deficiencies in this system (13). However, the consequences of disrupting angiotensin II activity, given its vast array of cell targets, are likely to be many, and evidence exists that the renin-angiotensin system can directly regulate macrophage function $(14,15)$. To more precisely examine the role of infiltrating macrophages in renal fibrogenesis, Nishida et al. (11) used wild-type mice that were transplanted, after lethal irradiation, with bone marrow from mice lacking the Agtr1a gene. Unexpectedly, 2 weeks after UUO, kidneys from mice with Agtr1-deficient bone marrow showed more renal fibrosis and less infiltrating macrophages than did those from wildtype mice. Agtr1a-deficient mice also had less circulating monocytes and less macrophage precursors in the bone marrow. 
Two exciting conclusions can be drawn from this work. First, it suggests that, much indirect evidence notwithstanding, infiltrating macrophages activated by angiotensin can have antifibrotic action in the kidney; and second, it highlights the potentially important interaction between bone marrow cells and the kidney. How could infiltrating macrophages have antifibrotic action? Given the myriad actions described for these cells, the possibilities are many, but it could simply be due to their ability to phagocytize dead cells and cellular debris. Indeed, analysis of macrophage function by Nishida et al. (11) revealed that macrophages lacking Agtr1a have reduced migratory capacity and decreased phagocytic activity, a characteristic that could also be elicited in wild-type macrophages by the angiotensin II receptor antagonist losartan. Since inhibitors of the renin-angiotensin system are widely used in clinical medicine, it is urgently important to determine whether they block beneficial effects of invading macrophages. Experiments that reverse the model of Nishida et al. (11), i.e., UUO in mice that lack Agtr1a but contain wild-type bone marrow, should answer this question.

Nishida et al. (11) consider other possibilities by which invading macrophages could inhibit renal fibrosis. Macrophages can be a source of HGF (16), recently found to block the conversion of renal epithelia to mesenchymal cells $(17,18)$. However, because the presence of smooth muscle actin (a marker for renal interstitial fibroblasts) in the chimeras was similar to that in wild-type mice, the authors suggest that this conversion was unchanged in the chimeras. Another intriguing possibility they consider is that generation of reparative capillaries depends on invading macrophages, well known to secrete angiogenic factors. Capillary density quantified by CD31 was, surprisingly, no different between the two sets of animals. However, because newly generated endothelial cells may be negative for this marker (19), and Agtr1 appears to be involved in ischemia-induced angiogenesis (20), this possibility needs to be kept in mind.
Other, more speculative possibilities may be considered. Because pathological changes in the kidney can be selfrepaired and not result in renal fibrosis, adult kidneys may contain organ-specific stem cells, and invading macrophages (or other cells from the bone marrow) may provide critical signaling for their activation. Needless to say, quite independently of macrophages, the bone marrow could also be a source of cells that directly contribute to kidney repair. A variety of exciting reports have suggested that bone marrow may function as a reservoir of stem cells for adult organs (21, 22) suggesting that hematopoietic stem cells possess broad developmental plasticity that may be important for tissue repair. While recent work indicates that under normal conditions the developmental potential of the hematopoietic stem cell may be more restricted than originally believed (23), it may be different in diseases where selective pressure may amplify rare stochastic events. Furthermore, bone marrow also contains mesenchymal stem cells that might migrate to disease organs and participate in tissue repair (24). Regardless of the mechanism whereby invading macrophages (and/or other bone marrow cells) exert their antifibrotic effect, Nishida et al. (11) cast a new light on the role of macrophages in renal fibrosis and, more importantly, call attention to possible interactions between bone marrow cells and the kidney. Indeed, the kidneys of rats transplanted with bone marrow expressing green fluorescent protein (GFP) show a surprising abundance of interstitial GFP-positive cells (25), further supporting the notion that bone marrow cells play important and unexpected roles in renal regulation and disease.

1. Anderson, S., et al. 1985. Control of glomerular hypertension limits glomerular injury in rats with reduced renal mass. J. Clin. Invest. 76:612-619.

2. Taal, M.W., and Brenner, B.M. 2000. Renoprotective benefits of RAS inhibition: from ACEI to angiotensin II antagonists. Kidney Int. 57:1803-1817.

3. Border, W.A., et al. 1990. Suppression of experimental glomerulonephritis by antiserum against transforming growth factor beta 1 . Nature. 346:371-374

4. Yamamoto, T., et al. 1994. Sustained expression of TGF-beta 1 underlies development of progressive kidney fibrosis. Kidney Int. 45:916-927.
5. Iwano, M., et al. 2002. Evidence that fibroblasts derive from epithelium during tissue fibrosis. J. Clin. Invest. 110:341-350. doi:10.1172/JCI200215518.

6. Barasch, J., et al. 1999. Mesenchymal to epithelial conversion in rat metanephros is induced by LIF. Cell. 99:377-386.

7. Holdsworth, S.R, Neale, T.J., and Wilson, C.B. 1981 Abrogation of macrophage-dependent injury in experimental glomerulonephritis in the rabbit. Use of an antimacrophage serum. J. Clin. Invest. 68:686-698.

8. Tesch, G.H., et al. 1999. Monocyte chemoattractant protein-1 promotes macrophage-mediated tubular injury, but not glomerular injury, in nephrotoxic serum nephritis. J. Clin. Invest. 103:73-80.

9. Ophascharoensuk, V., et al. 1999. Obstructive uropathy in the mouse: role of osteopontin in interstitial fibrosis and apoptosis. Kidney Int. 56:571-580.

10. Lange-Sperandio, B., et al. 2002. Selectins mediate macrophage infiltration in obstructive nephropathy in newborn mice. Kidney Int. 61:516-524.

11. Nishida, M., et al. 2002. Absence of angiotensin II type 1 receptor in bone marrow-derived cells is detrimental in the evolution of renal fibrosis. J. Clin. Invest. 110:1859-1868. doi:10.1172/JCI200215045.

12. Satoh, M., et al. 2001. Renal interstitial fibrosis is reduced in angiotensin II type 1a receptor-deficient mice. J. Am. Soc. Nephrol. 12:317-325.

13. Fern, R.J., et al. 1999. Reduced angiotensinogen expression attenuates renal interstitial fibrosis in obstructive nephropathy in mice. J. Clin. Invest. 103:39-46.

14. Weinstock, J.V., and Kassab, J.T. 1984. Angiotensin II stimulation of granuloma macrophage phagocytosis and actin polymerization in murine schistosomiasis mansoni. Cell. Immunol. 89:46-54.

15. Scheidegger, K.J., Butler, S., and Witztum, J.L. 1997 Angiotensin II increases macrophage-mediated modification of low density lipoprotein via a lipoxygenase-dependent pathway. J. Biol. Chem 272:21609-21665.

16. Chen, Q., DeFrances, M.C., and Zarnegar, R. 1996. Induction of met proto-oncogene (hepatocyte growth factor receptor) expression during human monocyte-macrophage differentiation. Cell Growth Differ. 7:821-832

17. Mizuno, S., et al. 1998. Hepatocyte growth factor prevents renal fibrosis and dysfunction in a mouse model of chronic renal disease. J. Clin. Invest. 101:1827-1834.

18. Yang, J., and Liu, Y. 2002. Blockage of tubular epithelial to myofibroblast transition by hepatocyte growth factor prevents renal interstitial fibrosis. J. Am. Soc. Nephrol. 13:96-107.

19. Oliver, J.A., Goldberg, M.R., and Al-Awqati, Q. 1997. Endothelial cell targeting during renal development: use of monoclonal antibodies. Am. J. Physiol. 272:F153-F159.

20. Sasaki, K., et al. 2002. Evidence for the importance of angiotensin II type 1 receptor in ischemia-induced angiogenesis. J. Clin. Invest. 109:603-611. doi:10.1172/JCI200213055.

21. Petersen, B.E., et al. 1999. Bone marrow as a potential source of hepatic oval cells. Science. 284:1168-1170.

22. Gussoni, E., et al. 1999. Dystrophin expression in the $\mathrm{mdx}$ mouse restored by stem cell transplantation. Nature. 401:390-394.

23. Wagers, A.J., et al. 2002. Little evidence for developmental plasticity of adult hematopoietic stem cells. Science. 297:2256-2259.

24. Pittenger, M.F., et al. 1999. Multilineage potential of adult human mesenchymal stem cells. Science. 284:143-147.

25. Ito, T., et al. 2001. Application of bone marrowderived stem cells in experimental nephrology. Exp. Nephrol. 9:444-450. 